\title{
Optimal sample size calculation for null hypothesis significance tests
}

$4 \quad{ }^{1}$ Business Intelligence Department, Co-operators General Insurance Company, 130 Macdonell

5 St., Guelph, Ontario, N1H 2P6

$6{ }^{2}$ Department of Biological Sciences, The Canadian Rivers Institute, 100 Tucker Park Road,

7 University of New Brunswick, Saint John, New Brunswick, E2L 4L5

8

9 Corresponding author: Jeff Houlahan; email - jeffhoul@unb.ca; phone - 


\section{Abstract}

Traditional study design tools for estimating appropriate sample sizes are not consistently used in ecology and can lead to low statistical power to detect biologically relevant effects. We have developed a new approach to estimating optimal sample sizes, requiring only three parameters; a maximum acceptable average of $\alpha$ and $\beta$, a critical effect size of minimum biological relevance, and an estimate of the relative costs of Type I vs. Type II errors. This approach can be used to show the general circumstances under which different combinations of critical effect sizes and maximum acceptable combinations of $\alpha$ and $\beta$ are attainable for different statistical tests. The optimal $\alpha$ sample size estimation approach can require fewer samples than traditional sample size estimation methods when costs of Type I and II errors are assumed to be equal but recommends comparatively more samples for increasingly unequal Type I vs. known, optimal sample size estimation can be used to determine the smallest sample size at which the cost of an additional sample outweighs its associated reduction in errors. Optimal sample size estimation constitutes a more flexible and intuitive tool than traditional sample size estimation approaches, given the constraints and unknowns commonly faced by ecologists during study. 


\section{Introduction}

Good study design ensures that scientists can make meaningful and appropriate inferences from data. The influence of sample size on inference strength has been well documented in ecology for species richness estimates (Heck et al. 1975), species distributions (Hernandez et al. 2006) and genetic diversity (Leberg 2002). In addition to affecting the precision of parameter estimates, sample size has a major impact on the statistical power of a null hypothesis significance test to detect an effect (Steidl et al. 1997, Jennions and Moller 2003).

Techniques exist for estimating appropriate numbers of samples for most null hypothesis significance tests (Zar 2007). These techniques estimate the minimum sample size needed to achieve the desired power $(1-\beta)$ to detect the critical effect size, given the expected variability and the chosen $\alpha$ level. Thus, they require specification of $\alpha, \beta$, critical effect size and expected variability. All too often these tools go unused (or at least unreported) in ecological research, especially in field-based studies. The reasons for this include that (1) often the replication required to meet accepted power levels is either logistically impractical or prohibitively expensive and sample size may often be restricted by funding, time, space, regulatory, and/or personnel constraints, (2) a number of the parameters in sample size estimation formulas, including critical effect sizes, acceptable $\alpha$ and $\beta$ levels and even expected variability, are difficult for ecologists to estimate a priori, and (3) ecologists don't give statistical power the consideration is deserves.

Scientists have reached a somewhat arbitrary consensus on the acceptable $\alpha$ level (i.e.Type I error rate) under the null hypothesis $(\alpha=0.05)$. Despite this consensus, when expected variability is high and/or biologically relevant effect sizes are small the use of $\alpha=0.05$ can require unattainably large numbers of samples. By contrast, there is no consensus on the 
70 acceptable $\beta$ level (i.e.Type II error rate) under the alternate hypothesis. Suggestions for

71 minimum acceptable levels of statistical power include $80 \%, 90 \%$ and $95 \%$ (i. e. Type I error rate

$72=$ Type II error rate). The result is that researchers may forego or ignore sample size estimation

73 and tolerate unreasonably high Type II error probabilities.

74 Identifying biologically relevant (i.e. critical) effect sizes is difficult and often subjective

75 (Munkittrick et al. 2009). This has led to the standard practice of only evaluating biological

76 significance after having checked for statistical significance (and sometimes erroneously

77 equating statistical significance, or lack thereof, with biological significance) (Martínez-Abraín

78 2008).

Further, estimation of the expected amount of variability among replicates is also

80 frequently difficult for ecologists. Pilot studies intended to estimate variability levels for sample

81 size estimation can have the same logistical and financial constraints as noted above for the

82 primary study. Further, variability estimates from previously published studies may be unreliable

83 because variability in natural systems often differs among species, locations and times. This has

84 led researchers to invest in methodological techniques that limit variability and thus increase the

85 power to detect effects (Munkittrick et al. 2009).

Mudge et al. (2012) describes an approach for setting optimal $\alpha$ levels for null hypothesis

87 significance tests. This approach minimizes either the combined probabilities or costs of Type I

88 and II errors given a specific combination of critical effect size, relative costs of Type I vs. Type

89 II error and relative prior probabilities of null and alternate hypotheses. Instead of specifying the

90 sample size (among other things) to calculate the smallest possible combination of $\alpha$ and $\beta$, the

91 approach can be modified to allow an optimal sample size calculation, given a maximum

92 acceptable combination of $\alpha$ and $\beta$. 
If the sample size recommendation based on preliminary acceptable error rates and target

94 effect sizes are impractical, researchers would be able to modify either the amount of error they

95 are willing to accept, modify the critical effect size relative to the variability that they wish to be

96 able to detect, or modify the endpoint and/or study design. This approach forces researchers to be

97 explicit about what effects sizes they are targeting and what error rates they are willing to accept

98 (rather than using default Type I error rates and arbitrary Type II error rates). Further, once

99 those decisions are made it allows researchers to identify the minimum number of required

100 samples. This would allow researchers, on the one hand, to avoid doing more sampling than

101 necessary and, on the other, to avoid doing research that doesn't have the power to detect

102 relevant effect sizes with an acceptable error rate.

103 Our objectives are to (1) describe how the optimal $\alpha$ approach can be used to calculate

104 optimal (i.e. minimum) sample sizes during study design, (2) show how optimal sample size is

105 affected by the maximum acceptable average of $\alpha$ and $\beta$, critical effect size, and relative costs of

106 Type I vs. Type II errors, (3) compare and contrast sample size estimates made using optimal $\alpha$

107 to traditional sample size estimation approaches, and (4) provide example scenarios that

108 demonstrate how estimating optimal sample sizes could be applied in ecology research.

109

110

111

112

113

114

115 combination of sample size, critical effect size, relative costs of Type I vs. Type II error and

116 relative prior probabilities of null vs. alternate hypotheses, there exists a single combination of $\alpha$

117 and $\beta$ that minimizes the average of Type I and Type II errors. Similarly, an optimal sample size 
118 can be estimated because for any combination of desired Type I and II error rates, critical effect

119 size, relative costs of Type I vs. Type II error and relative prior probabilities of null vs. alternate

120 hypotheses, there exists a minimum sample size that can achieve the targeted Type I and Type II

121 error rates. (Note, to simplify the explanation we have assumed that the prior probability of Ho

122 and Ha are equal. However, optimal sample size can be calculated when that assumption isn't

123 met). Optimal significance levels that minimize combined probabilities of Type I and II errors in

124 null hypothesis tests can be calculated through an iterative process of examining the average of $\alpha$

125 and $\beta$ over $\alpha$ values ranging from 0 to 1 , to determine the $\alpha$ value that results in the smallest

126 average of $\alpha$ and $\beta$. Similarly, for a given critical effect size and relative costs of Type I vs. Type

127 II errors, optimal sample size can be calculated through another iterative process that examines

128 the average of the optimal $\alpha$ and the associated $\beta$ over a range of sample sizes to iteratively

129 determine the smallest sample size at which the average of $\alpha$ and $\beta$ remains below the maximum

130 acceptable average of $\alpha$ and $\beta$.

131 Optimal sample sizes will differ for each combination of critical effect size, maximum

132 acceptable average of $\alpha$ and $\beta$, relative (or absolute) costs of Type I vs. Type II errors and test

133 type.

134 Simulations

135

To examine how optimal sample size is influenced by other aforementioned parameters,

137 we have begun with a simple, two-tailed, two-sample independent t-test and plotted the optimal

138 sample size at different combinations of critical effect size and maximum acceptable average of

$139 \alpha$ and $\beta$, assuming equal costs of Type I vs. Type II error. We then show examples of the optimal

140 sample sizes for the same test-type and the same ranges of critical effect size and maximum

141 acceptable averages of $\alpha$ and $\beta$, under four different unequal relative costs of errors to show how 
142 optimal sample size changes when either Type I errors are considered to be either $0.1 \mathrm{x}, 0.5 \mathrm{x}, 2 \mathrm{x}$

143 or 10x the cost of Type II errors. Then, we return to assuming equal relative costs of Type I and

144 Type II errors and contrast the optimal sample sizes recommended at different combinations of

145 critical effect size and maximum acceptable average of $\alpha$ and $\beta$ for simple linear correlations and

146 a one-way ANOVA with 2, 4, 6 and 8 treatment groups.

147 To directly compare sample size recommendations generated using the optimal $\alpha$

148 approach with sample sizes generated using the traditional sample size estimation technique that

149 requires specifying desired $\alpha$ and $\beta$ levels, we chose a two-tailed, two-sample t-test over a range

150 of critical effect sizes and relative costs of Type I vs. Type II error and compared differences in

151 sample size recommendations between a standard approach targeting $\alpha=0.05$ and $\beta=0.2(80 \%$

152 statistical power) and the optimal $\alpha$ approach targeting a maximum acceptable average of $\alpha$ and $\beta$

153 of 0.125 , the value associated with using $\alpha=0.05$ and attempting to achieve $80 \%$ statistical

154 power.

\section{Scenarios}

We also present two hypothetical scenarios to demonstrate how optimal sample size calculation

162 can be used in applied ecology, with: (1) Absolute cost of errors not known and relative costs of 163 errors estimated, and (2) Absolute costs of errors estimated.

\section{Relative costs of errors estimated}

The first scenario involved testing for a relationship between the environmental

168 concentration of a chemical and species abundance at different sites, after the chemical has been 
169 observed to have a detrimental effect in a laboratory setting. A significant relationship would

170 trigger management actions to reduce chemical inputs to the environment. A Type I error would

171 result in unnecessary management action while a Type II error would result in unmitigated

172 impacts on the species of interest. Absolute costs of Type I and II errors are unknown but two

173 scenarios are presented: (1) Falsely concluding a relationship exists is 0.25 as serious as missing

174 a real relationship (cost of Type I error $=0.25^{*}$ cost of Type II error, as is loosely implied by

175 using $\alpha=0.05$ and $80 \%$ statistical power) and (2) detecting a false relationship is equally serious

176 as missing a real relationship. Two maximum averages of $\alpha$ and $\beta$ are presented: (1) 0.01 where

177 errors, in general, are serious and (2) 0.1 where errors are less serious, e.g. if the species is less

178 valuable and management actions are less expensive. Two critical effect sizes at which

179 management action would be warranted are presented, (1) a relationship where the chemical

180 explains half of the variance in abundance counts (i.e. $\left.\mathrm{R}^{2} \geq 0.5\right)$ and (2) a relationship where the

181 chemical explains at least one quarter of the variance in abundance counts (i.e. $\mathrm{R}^{2} \geq 0.25$ ). The

182 resulting combinations of sample size calculation parameters are summarized in Error!

183 Reference source not found..

184 Figure 1: Combinations of relative error costs, acceptable averages of $\alpha$ and $\beta$, and critical effect sizes

185 examined for the relative error costs sample size scenario.

186

187

188

189

190 191 required, while a non-significant result would signify that the population is not significantly

192 greater than its minimum viable size and would trigger conservation management actions. In this

\section{Absolute costs of errors estimated}

The second scenario involves monitoring to see if species abundance is significantly greater than the estimate of minimum viable population size. A significant result would signify that the population is well above the minimum viable size and that no management action is 
193 case, a Type I error would result in managers being unaware that a population is potentially

194 falling below its minimum viable size, while a Type II error would result in unwarranted

195 conservation management actions. Two absolute costs of errors are examined, one where the

196 focal species is economically valuable so a Type I error will cost ten times more than a Type II

197 error (at \$10 000000 vs. \$1 000000 respectively) and another where the cost of conservation is

198 equivalent to the value of the species (at $\$ 1000000$ each). Two potential sample costs were

199 examined, $\$ 1000$ and $\$ 10000$ per sample. Also presented are results for two potential critical

200 effect sizes at which proximity to the minimum viable population would trigger conservation

201 management: one and two standard deviations from the minimum viable population size. Note

202 that we don't set a maximum average error rate because the average error rate is decided by the

203 sample size at which the cost of adding an additional sample would not reduce the costs of an

204 error enough to offset the cost of an additional sample. For example, if the costs of Type I and II

205 errors were each $\$ 1,000,000$ dollars and the cost of an additional sample was $\$ 1,000$ we would

206 only add an additional sample if it reduced the average error rate by more than 0.001 , The

207 resulting combinations of sample size calculation parameters are summarized in Fig. 2.

Figure 2: Combinations of absolute error costs, sampling costs, and critical effect sizes examined for the

absolute error costs sample size scenario.

Results

211

\section{Simulations}

t-test: equal costs of errors

217 average of $\alpha$ and $\beta$ decrease (Fig. 3). For a 2-tailed, 2-sample t-test with equal numbers of 218 samples in each group and equal relative costs of Type I vs. Type II error, fewer than 15 samples 
219 are required to detect critical effect sizes $\geq 2$ standard deviations of the data at all but the smallest

220 maximum acceptable averages of $\alpha$ and $\beta$. By contrast, optimal sample size recommendations are

221 larger than15 for a critical effect size of 1 standard deviation of the data for any maximum

222 acceptable average of $\alpha$ and $\beta>0.05$. More than 30 samples are required to detect effects $\leq 0.5$

223 SD for all maximum acceptable averages of $\alpha$ and $\beta<0.15$. At any given maximum acceptable

224 average of $\alpha$ and $\beta$, the relationship between critical effect size and optimal sample size is

225 nonlinear, requiring increasingly large increases in sample size as critical effect size decreases.

226 For example, if we set the maximum acceptable average of $\alpha$ and $\beta$ at 0.125 , halving the critical

227 effect size from 1.0 to 0.5 SD more than triples the optimal sample size (Fig. 4).

228 Figure 3: The minimum number of samples in each group that are required to achieve the desired maximum

229 acceptable average of Type I and Type II error rates over a range of critical effect sizes (expressed in

230 standard deviation units), for a 2-tailed, 2-sample t-test with an equal number of samples in each group and

231 equal relative costs of Type I vs. Type II error.

232 Figure 4: The influence of the choice of critical effect size (expressed in standard deviation units) on the

233 optimal sample size for a two-tailed, two-sample t-test, assuming equal relative costs of Type I and II errors

234 and holding the maximum acceptable average of $\alpha$ and $\beta$ at 0.125 .

t-test: unequal costs of errors

When relative costs of Type I and Type II error are unequal, larger sample sizes are required

238 to detect the same combinations of critical effect size and maximum acceptable average of $\alpha$ and

$239 \beta$, relative to those recommended for equal relative costs of Type I and II errors (Fig. 5) and the

240 greater the deviation from equality of the relative costs of Type I and II errors, the greater the

241 increase in required sample size.However, the influence of relative error costs on sample size

242 recommendation is much smaller than the influence of either critical effect size or maximum 
243 acceptable average of $\alpha$ and $\beta$. There are also marginal differences in recommended sample size

244 depending on which error type is more costly.

245 Figure 5: The minimum number of samples in each group that are required to achieve the desired maximum

246 acceptable average of Type I and Type II error rates over a range of critical effect sizes (expressed as

247 standard deviation units), for a 2-tailed, 2-sample t-test with an equal number of samples in each group and

248 (A) Type I errors considered half as serious as Type II errors, (B) Type I errors considered twice as serious as

249 Type II errors (C) Type I errors considered 10 times less serious than Type II errors and (D) Type I errors

250 considered 10 times more serious than Type II errors.

\section{Correlation and ANOVA tests}

254 size recommendation differ among test types (Fig. 6, Fig. 7). For simple linear correlation tests

255 (Fig. 6), sample sizes smaller than 10 are rarely informative, as these small sample sizes are only

256 recommended when either the critical effect size is $\geq r=0.7\left(\mathrm{R}^{2}=0.5\right)$ and/or the maximum

257 acceptable average of $\alpha$ and $\beta$ is $\geq 0.2$. Sample sizes approaching 30 can be more informative,

258 with these sample sizes being recommended when either the critical effect size is $r=0.5\left(R^{2}=\right.$

2590.25 ) and/or the maximum acceptable average of $\alpha$ and $\beta$ is $\geq 0.125$. In ANOVA study designs

260 (Fig. 7), increasing the number of levels of a factor decreases the number of replicates required

261 within each level of the factor. However, this decrease in the number of replicates required

262 within each factor level comes at the expense of an even greater increase in the total number of

263 replicates required among all factor levels, such that using more replicates within fewer factor

264 levels is more efficient than using fewer replicates within more factor levels. The total number of

265 samples in an ANOVA design required to achieve a maximum acceptable average of $\alpha$ and $\beta$ of

2660.05 for a critical effect size of $\mathrm{f}^{2}=1$ is 16 with 2 groups ( 8 replicates $* 2$ groups), 24 with 4 
groups ( 6 replicates $* 4$ groups), 30 with 6 groups ( 5 replicates $* 6$ groups), and 32 with 8 groups

268 (4 replicates $* 8$ groups).

Figure 6: The minimum number of samples that are required to achieve the desired maximum acceptable average of Type I and Type II error rates over a range of critical effect sizes (expressed as a correlation coefficient), for a 2-tailed simple linear correlation test with equal relative costs of Type I vs. Type II error.

272 Figure 7: The minimum number of replicates in each group that are required to achieve the desired

273 maximum acceptable average of Type I and Type II error rates over a range of critical effect sizes (expressed 274 as a ratio of among-group variance to within-group variance), for a one-way ANOVA with an equal number 275 of replicates in each group, equal relative costs of Type I vs. Type II error and (A) 2 treatment groups, (B) 4 276 treatment groups (C) 6 treatment groups and (D) 8 treatment groups

t-test: comparison between optimal $\alpha$ and traditional sample size recommendations

280 Type I and II errors deviate from equality. The traditional approach of designing t-test studies to 281 achieve $80 \%$ statistical power with an $\alpha=0.05$ also results in larger sample size

282 recommendations as critical effect size decreases, however this approach does not recommend 283 adjusting sample sizes when relative costs of Type I and II errors differ. When relative costs of

284 Type I and II errors are equal, the optimal $\alpha$ sample size estimation approach can achieve the 285 same maximum acceptable average of $\alpha$ and $\beta$ at the same effect size using fewer samples than 286 the traditional sample size estimation approach using $\alpha=0.05$ and $80 \%$ statistical power (Fig. 8).

287 As relative costs of Type I vs. Type II errors become more unequal, the sample sizes

288 recommended by the optimal $\alpha$ approach increase to the point where they surpass those

289 recommended by the traditional sample size estimation approach using $\alpha=0.05$ and $80 \%$

290 statistical power. Optimal $\alpha$ sample size recommendations are larger than those recommended by

291 traditional sample size estimation approach for highly unequal relative costs of Type I vs. Type 
292 II errors, however when relative costs of Type I and II errors are highly unequal, the practice of

293 using $\alpha=0.05$ and $80 \%$ statistical power becomes less and less justifiable.

294 Figure 8: Comparison of the sample size recommendations between the optimal $\alpha$ approach (shaded areas)

295 and the traditional sample size estimation approach (horizontal lines), over a range of critical effect sizes

296 (expressed in standard deviation units) and relative costs of Type I vs. Type II errors, for a two-tailed, two-

297 sample t-test using a maximum acceptable average of $\alpha$ and $\beta$ of 0.125 for the optimal $\alpha$ approach and $\alpha=0.05$

298 and $\boldsymbol{\beta}=\mathbf{0 . 2}$ for the traditional sample size estimation approach.

\section{Scenarios}

Absolute costs of errors unknown

304 of Type I vs. Type II error cost ratio, maximum acceptable average of $\alpha$ and $\beta$, and critical effect

305 size (Table 1). Decreasing the Type I vs. Type II error cost from 1 to 0.25 resulted in small

306 increases in sample size recommendations of 2 to 6 samples. A 10-fold decrease in the maximum

307 acceptable average of $\alpha$ and $\beta$, and a 2-fold decrease in critical effect size each resulted in a

308 greater than 2-fold increase in the sample size recommendation.

309 Table 1: Optimal sample sizes that achieve a maximum acceptable average of $\alpha$ and $\beta$ for a simple linear

310 regression under different relative costs of Type I vs. Type II error, maximum acceptable averages of $\alpha$ and $\beta$

311 and critical effect sizes, with associated $\alpha$ and $\beta$ levels for the optimal sample size.

\begin{tabular}{cccccc}
\hline $\begin{array}{c}\text { Type I vs. Type } \\
\text { II error cost } \\
\text { ratio }\end{array}$ & $\begin{array}{c}\text { Maximum } \\
\text { acceptable average } \\
\text { of } \alpha \text { and } \beta\end{array}$ & $\begin{array}{c}\text { Critical effect } \\
\text { size }\left(R^{2}\right)\end{array}$ & $\begin{array}{c}\text { Optimal } \\
\text { sample } \\
\text { size }\end{array}$ & $\begin{array}{c}\text { Optimal } \\
\alpha\end{array}$ & $\begin{array}{c}\text { Optimal } \\
\beta\end{array}$ \\
\hline 1 & 0.01 & 0.5 & 34 & 0.010 & 0.010 \\
1 & 0.01 & 0.25 & 83 & 0.009 & 0.010 \\
1 & 0.1 & 0.5 & 14 & 0.091 & 0.103 \\
1 & 0.1 & 0.25 & 31 & 0.091 & 0.108 \\
0.25 & 0.01 & 0.5 & 37 & 0.015 & 0.003 \\
0.25 & 0.01 & 0.25 & 89 & 0.016 & 0.004 \\
& & & & &
\end{tabular}




\begin{tabular}{llllll}
0.25 & 0.1 & 0.5 & 16 & 0.166 & 0.033 \\
0.25 & 0.1 & 0.25 & 37 & 0.164 & 0.033 \\
\hline
\end{tabular}

Absolute costs estimated

314

Optimal sample size recommendations ranged from 6 to 36, depending on the combination of

316 Type I error cost, cost per sample, and critical effect size (Table 2). Increasing the cost of Type I

317 errors from $\$ 1000000$ to $\$ 10000000$ resulted in sample size recommendations increasing by 4-

31810 samples. A 10 -fold decrease in the cost per sample and a 2-fold decrease in the critical effect

319 size each had large impacts on recommended sample size, each increasing the sample size

320 recommendation more than 2-fold. Total sampling costs were lowest when costs of errors were

321 equal, when the critical effect size was large and, as expected, when costs per sample were low.

\section{Discussion}

\section{Comparison between optimal and traditional sample size estimation}

327 seriousness of different potential errors and how much error they would be willing to accept as

328 central to good study design. The optimal $\alpha$ approach can directly incorporate each of these

329 important considerations in the calculation of either the minimum sample size that achieves the

330 maximum acceptable average of $\alpha$ and $\beta$, or the minimum sample size at which the cost of an

331 additional sample outweighs the reduction in errors associated with the additional sample. By

332 contrast, the traditional sample size estimation approach of calculating the minimum sample size

333 required to achieve a specific $\beta$ level fails to minimize the number of samples required.

334 Table 2: Optimal sample sizes at which the cost of an additional sample exceeds the reduction in error costs

335 for a one-tailed, one-sample t-test under different absolute error costs, sample costs and critical effect sizes,

336 with associated $\alpha$ and $\beta$ levels and the total sampling cost for the optimal sample size. 


\begin{tabular}{cccccccc}
\hline $\begin{array}{c}\text { Cost of Type } \\
\text { I error (\$) }\end{array}$ & $\begin{array}{c}\text { Cost of } \\
\text { Type II } \\
\text { error }(\$)\end{array}$ & $\begin{array}{c}\text { Cost per } \\
\text { sample } \\
(\$)\end{array}$ & $\begin{array}{c}\text { Critical } \\
\text { effect size } \\
\text { (Cohen's } \\
\text { d) }\end{array}$ & $\begin{array}{c}\text { Optimal } \\
\text { sample } \\
\text { size }\end{array}$ & $\begin{array}{c}\text { Optimal } \\
\alpha\end{array}$ & $\begin{array}{c}\text { Optimal } \\
\beta\end{array}$ & $\begin{array}{c}\text { Total } \\
\text { sampling } \\
\text { cost }(\$)\end{array}$ \\
\hline 1000000 & 1000000 & 1000 & 2 & 12 & 0.0033 & 0.0017 & 12000 \\
1000000 & 1000000 & 1000 & 1 & 26 & 0.0092 & 0.0072 & 26000 \\
1000000 & 1000000 & 10000 & 2 & 6 & 0.0341 & 0.0157 & 60000 \\
1000000 & 1000000 & 10000 & 1 & 10 & 0.0791 & 0.0587 & 100000 \\
10000000 & 1000000 & 1000 & 2 & 16 & 0.0003 & 0.0019 & 16000 \\
10000000 & 1000000 & 1000 & 1 & 36 & 0.0008 & 0.0080 & 36000 \\
10000000 & 1000000 & 10000 & 2 & 10 & 0.0027 & 0.0180 & 100000 \\
10000000 & 1000000 & 10000 & 1 & 18 & 0.0070 & 0.0809 & 180000 \\
\hline
\end{tabular}

\section{Critical effect sizes and sample size estimation}

340 The critical effect size chosen has a strong influence on the sample size recommendation. In

341 ecology, the small sample sizes that are frequently found in both lab and field experiments (i.e.

342 less than 10 samples) are only optimal for t-tests when critical effect sizes are moderate to large

$343(\geq 1.5 \mathrm{SD})$ (if we assume $((\alpha+\beta) / 2=0.125$, which occurs with $\alpha=0.05$ and $80 \%$ statistical

344 power). Many researchers may be unaware that they are making the implicit assumption that

345 small to moderate effects are not important. The larger sample sizes that are more frequently

346 found in observational studies (10-30 samples) have the capability to detect smaller effects in

347 terms relative to the within-group standard deviation ( $\geq 1 \mathrm{SD})$, however the within-group

348 standard deviation is often larger for observational studies than in comparatively more controlled

349 laboratory (or even field) experiments, meaning that a 1 SD effect represents a larger absolute

350 effect for observational studies than for tightly controlled experiments. Critical effect sizes of 0.5

351 SD or smaller require sample sizes that are rarely achieved in ecological research ( $>60$ samples).

352 In these situations, ecologists need to either 1) accept only being able to detect moderate to large 
353 effects, 2) tolerate larger averages of $\alpha$ and $\beta$ or 3) use more samples than is currently typical for

354 ecological research.

\section{Relative costs of Type I vs. II errors and sample size calculation}

The optimal $\alpha$ sample size approach shows that a few more samples should be used when one type of error is more serious than the other, relative to the number of samples recommended when Type I and II errors are equally serious. Relative costs of error are rarely addressed in ecological research and even less frequently are they incorporated into study design. The traditional approach to sample size estimation accepts whatever cost-ratio results in $\alpha=0.05$ and

$363 \beta=0.2$ at the researcher's chosen critical effect size. Note, that this does not correspond to a

364 Type I vs. Type II error cost ratio of 4, as assumed by Mapstone (1995), since the error

365 probability ratio and the error cost ratio are not the same (Mudge et al. 2012). Mapstone (1995) recommended setting $\alpha$ and $\beta$ so as to make $\beta / \alpha$ ratio equal to the Type I / Type II error cost ratio

367 both for sample size estimation and statistical analysis, however the flaw in this approach can be most easily illustrated when costs of Type I and II errors are equal. It may not be intuitively

369 obvious but, when $\alpha$ and $\beta$ are set to be equal to correspond with equal costs of errors, the outcome of shifting $\alpha$ and $\beta$ away from equality will virtually always yield a smaller average of $\alpha$

371 and $\beta$, and trading an increase in one error type for a greater corresponding reduction in the other

372 error type is always preferable when Type I and II errors have equal costs.

374 proposed by Field et al. (2004). Instead of attempting to minimize the probabilities or costs of

375 errors, this approach can be used to estimate sample sizes that minimize the total cost of

376 monitoring and management according to the probabilities and costs of errors. This approach to 
377 sample size estimation with a single goal of minimizing monitoring and management costs can

378 result in recommending a sample size of zero and essentially just assuming there either is an

379 effect or no effect when sampling costs are high and there is a large discrepancy between the

380 costs of Type I and II errors. While this approach may be valuable for applied environmental

381 management, it has received little attention in ecological research. In purely scientific research

382 the ultimate goal is accuracy of results, not cost-effectiveness so that, having low combined

383 probabilities of Type I and II errors is the main priority, and balancing Type I and II errors

384 appropriately according to their respective costs is of secondary concern.

\section{Differences among statistical test-types}

The relationship between the maximum acceptable average of $\alpha$ and $\beta$, critical effect size,

388 and optimal sample size does not remain consistent among statistical techniques. For

389 correlation/regression tests, the sample size recommendation curves on the graph of critical

390 effect size vs. maximum acceptable average of $\alpha$ and $\beta$ switch from curving outward away from

391 the origin to curving inward toward the origin at between 10 and 15 samples (Fig. 6). This means

392 that below 10 to 15 samples, correlation/regression tests are particularly ineffective for testing

393 null hypotheses as they are forced to choose between a very large critical effect size (for which

394 null hypothesis testing becomes increasingly unnecessary), or a very high maximum acceptable

395 average of $\alpha$ and $\beta$ (or both). For ANOVA, the sample size recommendation curves on the graph

396 of critical effect size vs. maximum acceptable average of $\alpha$ and $\beta$ have the same general shape as

397 for a t-test, with the key difference being that the curves shift downward as the number of

398 ANOVA factor levels increases, indicating that it takes fewer replicates per factor level to detect

399 effects of a given effect size as the number of factor levels increases. However, the total number

400 of samples required to detect a particular effect size will always be less with fewer factor levels. 
401 This means that to minimize sample sizes in ANOVA designs, one should use fewer factor levels

402 whenever possible. Other arguments for fewer factor levels in ANOVA designs are that ANOVA

403 results can be less informative as the number of factor levels increases (i.e. a result of significant

404 variability among many group means may not be surprising or useful for a large number of

405 groups), and the reliability of post-hoc pairwise comparison tests decreases as the number of

406 replicates within factor levels decreases.

\section{Maximum acceptable average of $\alpha$ and $\beta$ and sample size estimation}

410 that ought to concern researchers, funding/granting agencies, regulatory agencies and academic

411 publishers, but that is rarely dealt with directly. The most common combination of $\alpha$ and $\beta$ used

412 under the traditional sample size estimation approach is $\alpha=0.05$ and $\beta=0.2$ (i.e. $80 \%$ statistical

413 power), which yields an implied maximum acceptable average of $\alpha$ and $\beta$ of 0.125 . All else being

414 equal, a low average of $\alpha$ and $\beta$ is always better, as the average $\alpha$ and $\beta$ is an excellent indicator

415 of the quality of experimental design. Higher maximum acceptable averages of $\alpha$ and $\beta$ than

4160.125 may sometimes be appropriate, especially when samples are very expensive or difficult to

417 collect. One example of this is whole-system studies, which provide a level of realism that is

418 rarely attainable in smaller-scale studies with larger sample sizes. It is important to note that the

419 maximum upper threshold for maximum acceptable averages of $\alpha$ and $\beta$ would be anything

420 approaching 0.5 , the probability of reaching the correct conclusion using a coin toss. When

421 samples are cheap and easy to collect and/or when errors are very serious, a smaller average of $\alpha$

422 and $\beta$ than 0.125 may be warranted. Although the maximum acceptable average of $\alpha$ and $\beta$ is

423 something that is best evaluated on a study-specific basis, threshold maximum acceptable

424 averages of $\alpha$ and $\beta$ may sometimes be useful as one criterion for evaluation of grant 
application/renewal or as a component of the evaluation of manuscripts submitted for publication

426

427

428

429

430 in peer-reviewed scholarly journals. A suitable default maximum acceptable average of $\alpha$ and $\beta$ could be 0.05 , simply because we have become accustomed to the $5 \%$ value for Type I errors and confidence intervals. This would result in the default being 'tradition' but tradition used in a sensible way. Researchers should, however, start thinking more rigorously about what average of $\alpha$ and $\beta$ is acceptable, especially because using 0.05 as the default maximum acceptable average of $\alpha$ and $\beta$ is a more rigorous experimental design than the traditional approach of using $\alpha=0.05$ and $\beta=0.2$, and may require more samples to achieve.

\section{Conclusion}

The optimal $\alpha$ approach can be used to calculate optimal sample sizes during study design, recommending few samples when critical effect sizes are large, maximum acceptable averages of $\alpha$ and $\beta$ are large and relative costs of Type I vs. Type II errors are equal, and many samples at small critical effect sizes and maximum acceptable averages of $\alpha$ and $\beta$ and unequal relative costs of Type I vs. Type II errors. Holding the maximum acceptable average of $\alpha$ and $\beta$ constant, the optimal $\alpha$ approach recommended fewer samples than the traditional sample size estimation approach when relative costs of Type I and II errors were approximately equal, and more samples when relative costs were highly unequal. This is because the traditional approach is based on a fundamental assumption that relative costs aren't equal (i.e. $\alpha=0.05$ and $\beta=0.20$ ) so when the relative costs of error are equal, it is not as efficient as optimal $\alpha$. When the true relative costs are unequal the traditional approach recommends fewer samples but doesn't incorporate the true relative costs of error and so will not select the optimal number of samples. 
450 The flexibility of optimal sample size estimation allows for sample size estimates that achieve a

451 desired maximum acceptable average of $\alpha$ and $\beta$ given relative costs of Type I and II errors.

452 However, when absolute Type I and II error costs are known it also allows for sample size

453 estimates that identify when the cost of an additional sample would exceed the reduction in

454 overall error costs associated with adding the extra sample.

455 A brief guide to optimal sample size calculation

The first step in calculating sample sizes using the optimal $\alpha$ approach is to choose values

458 for the parameters on which the optimal sample size calculation will be based: the maximum

459 acceptable average of $\alpha$ and $\beta$, the relative costs of Type I and II errors, and the critical effect

460 size. Each of these parameters deserve rigorous consideration, however there are some sensible

461 default values that may be used in the absence of a strong rationale for any particular value.

462 Using 0.05 for the maximum acceptable average of $\alpha$ and $\beta$ would be consistent with the $5 \%$

463 standard with which we have become accustomed to using for Type I errors and confidence

464 intervals, while using 0.125 would be consistent with the common desire to achieve $80 \%$

465 statistical power at $\alpha 0.05$. For the relative costs of Type I and II errors, the most appropriate

466 default value (especially for purely scientific research) is to assume equal costs of Type I and II

467 errors, in favour of minimizing their combined probabilities. Although differences among

468 research questions precludes the potential for a default critical effect size that can be used for any

469 study design, see Nakagawa and Cuthill (2007) and Munkittrick et al. (2009) for discussions of

470 how to choose critical effect sizes that are associated with biological relevance.

471 Optimal sample sizes can be calculated for t-tests, ANOVA and regression/correlations

472 using code available online from Mudge et al. 2012.

\section{Authors Contributions}


$474 \mathrm{JM}$ and JH conceived the ideas. JM designed the methodology and simulations and took the lead

475 on writing. JM and JH contributed to drafts, interpretation of simulation results and approved

476 the final version.

477 Acknowledgements

We are grateful for Natural Sciences and Engineering Research Council funding.

\section{References}

Dupont P. Laplace and the indifference principle: Essai philosophique des probabilite's.

Field SA, Tyre AJ, Jonzen N, Rhodes JR, Possingham HP. Minimizing the cost of environmental management decisions by optimizing statistical thresholds. Ecol. Lett. 2004;7: 669-675.

Heck KL Jr., van Belle G, Simberloff D. Explicit calculation of the rarefaction diversity measurement and the determination of sufficient sample size. Ecology. 1975; 56: 14591461.

491 Hernandez PA, Graham CH, Master LL, Albert DL. The effect of sample size and species characteristics on performance of different species distribution modeling methods. Ecography. 2006; 29: 773-785.

494 Jennions MD, Moller AP. A survey of the statistical power of research in behavioral ecology and animal behavior. Behav. Ecol; 2003; 14: 438-445.

Leberg PL. Estimating allelic richness: Effects of sample size and bottlenecks. Mol. Ecol. 2002; 11: $2445-2449$.

498 Mapstone BD. Scalable decision rules for environmental impact studies: Effect size, Type I and Type II errors. Ecological Applications. 1995; 5: 401-410. 
500 Martínez-Abraín A. Statistical significance and biological relevance: A call for a more cautious

501 interpretation of results in ecology. Acta Oecolog. 2008; 34: 9-11.

502 Mudge JF, Baker LF, Edge CB, Houlahan JE. Setting an optimal $\alpha$ that minimizes errors in null

503 hypothesis significance tests. PLoS ONE. 2012; 7: e32734.

504 Munkittrick KR, Arens CJ, Lowell RB, Kaminski GP. A review of potential methods of

505 determining critical effect size for designing environmental monitoring programs.

$506 \quad$ Environ. Toxicol. Chem. 2009; 28: 1361-1371.

507 Nakagawa S, Cuthill IC. Effect size, confidence interval and statistical significance: a practical

508 guide for biologists. Biol. Rev. 2007; 82: 591-605.

509 Steidl RJ, Hayes JP, Schauber E. Statistical power analysis in wildlife research. J. Wild. Manag.

$510 \quad 1997 ; 61: 270-279$.

511 Zar J. Biostatistical Analysis. $5^{\text {th }}$ ed. Prentice-Hall, Inc. Upper Saddle River, NJ, USA. 942pp; $512 \quad 2007$. 


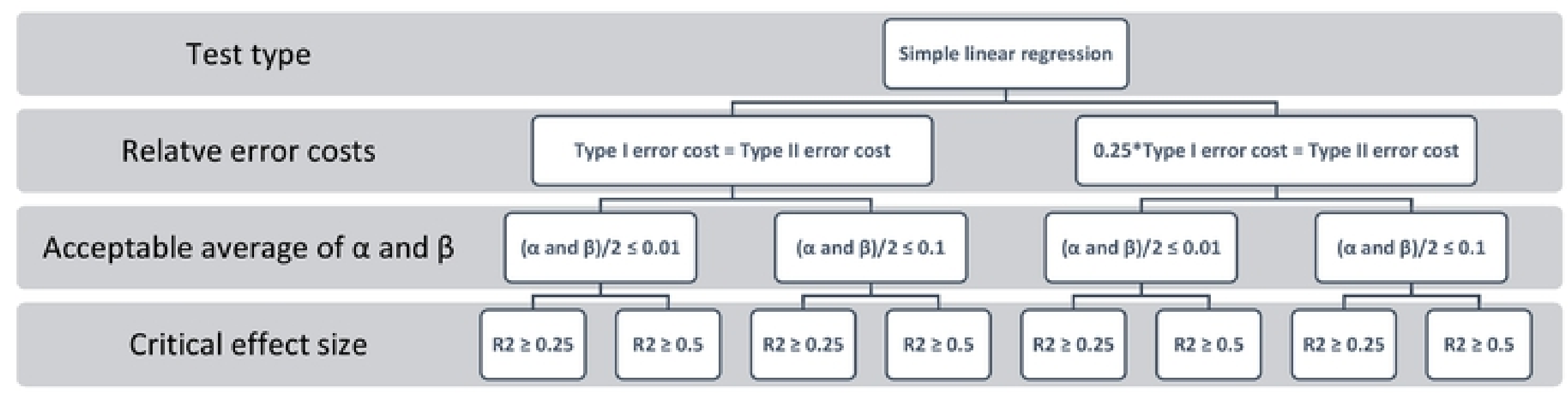

Figure 1 


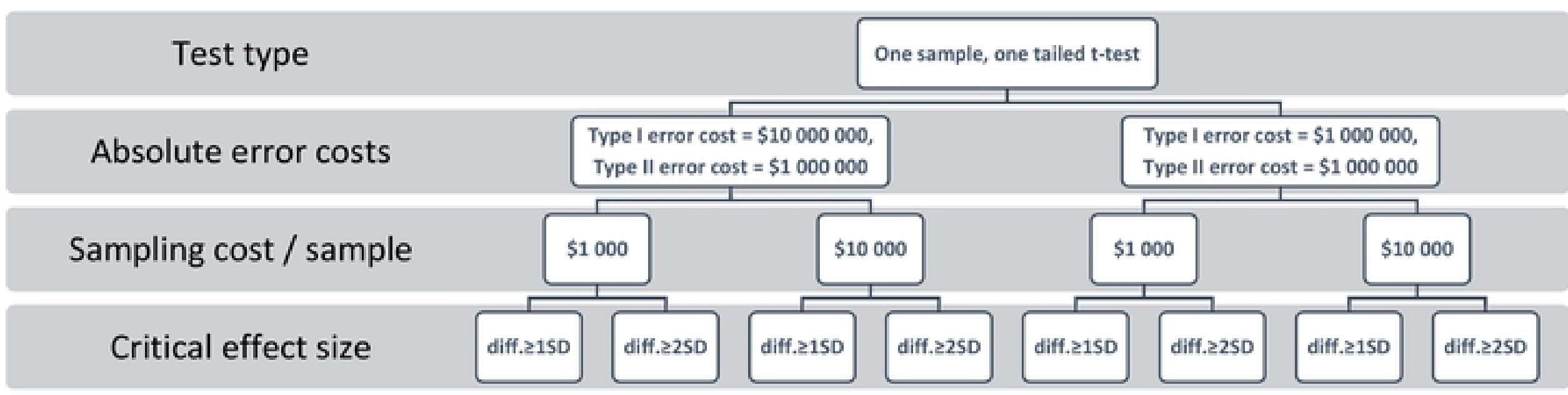

Figure 2 
Optimal
sample size

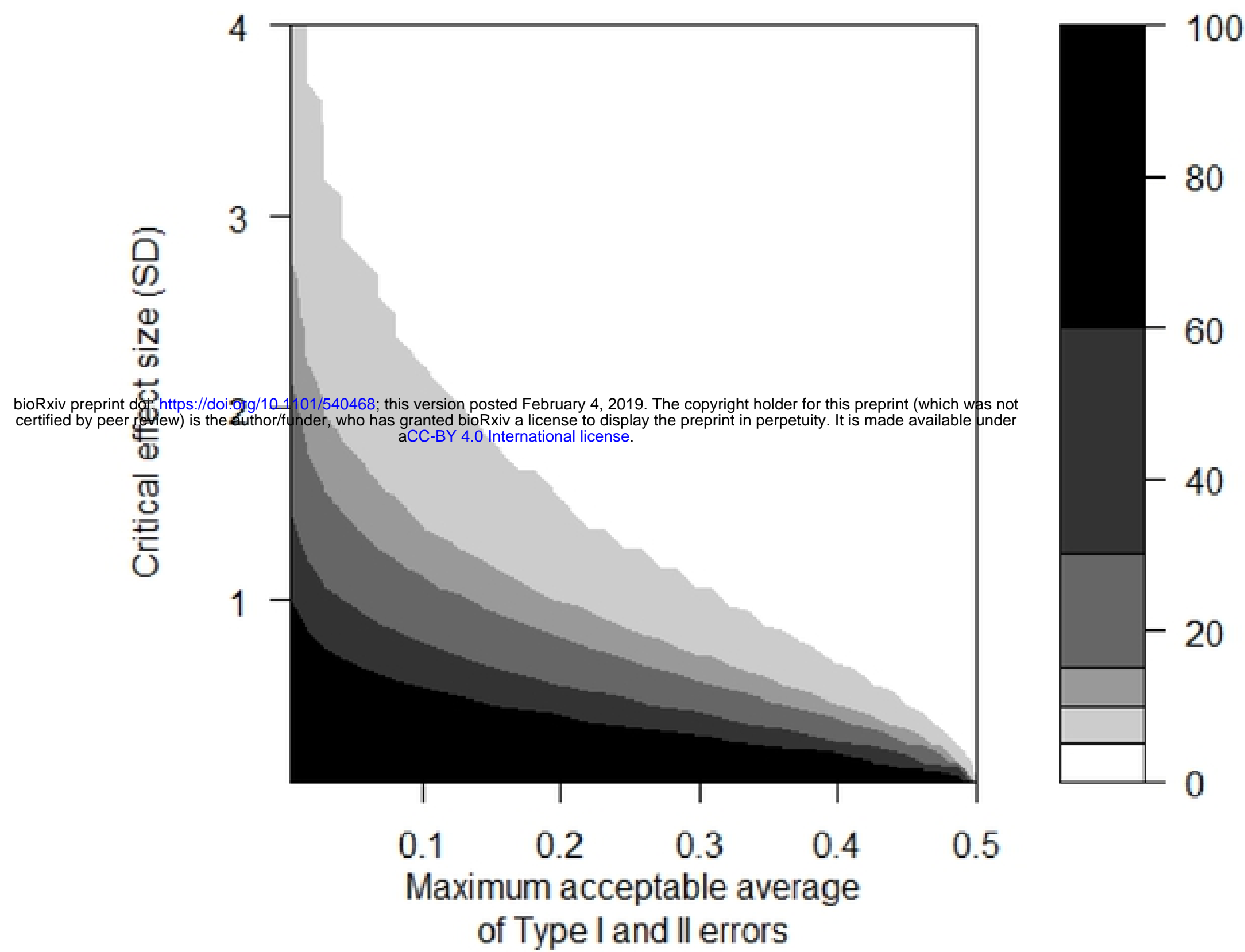

Figure 3 


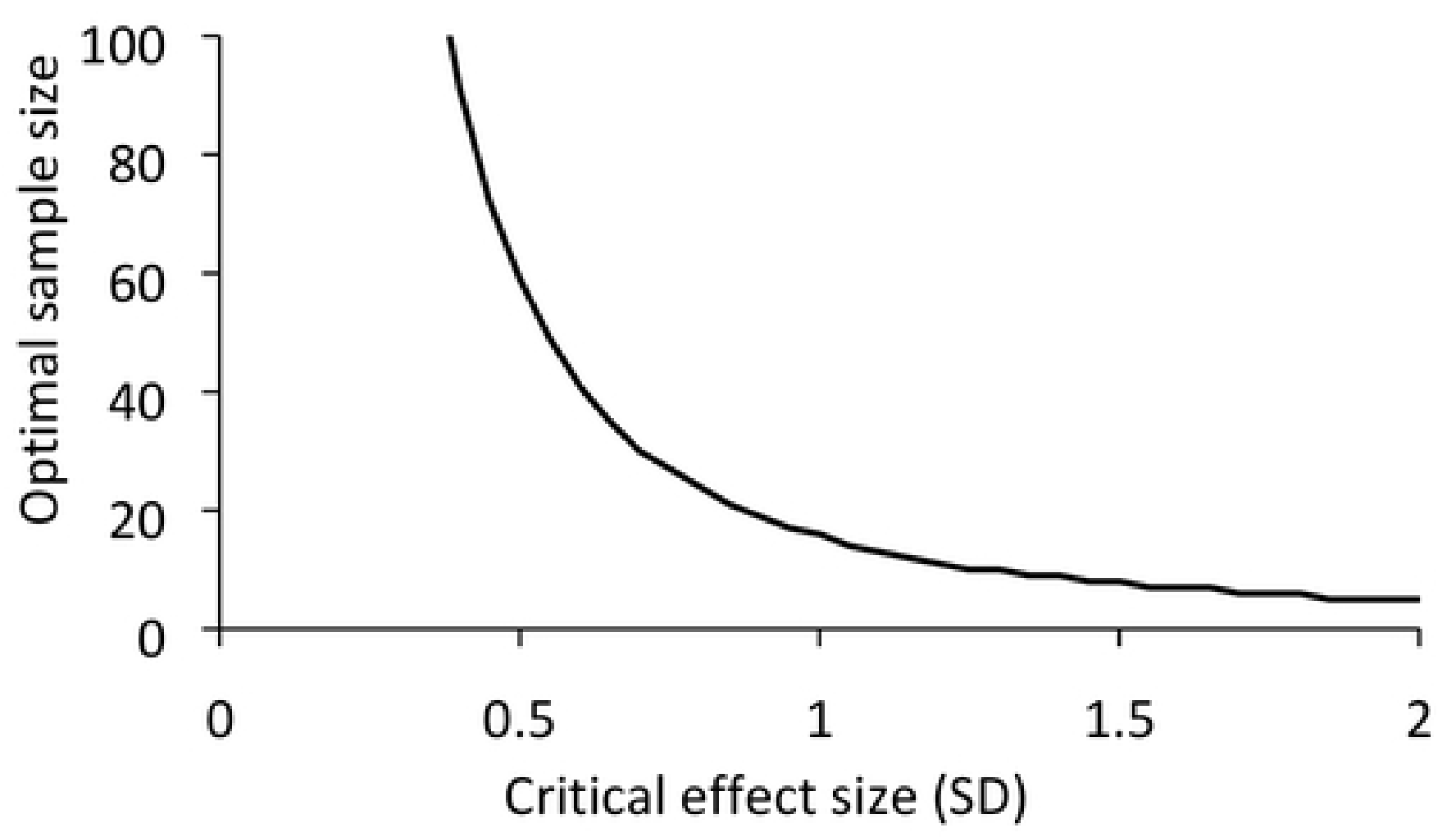

bioRxiv preprint doi: https://doi.org/10.1101/540468; this version posted February 4, 2019. The copyright holder for this preprint (which was not certified by peer review) is the author/funder, who has granted bioRxiv a license to display the preprint in perpetuity. It is made available under

Figure 4 

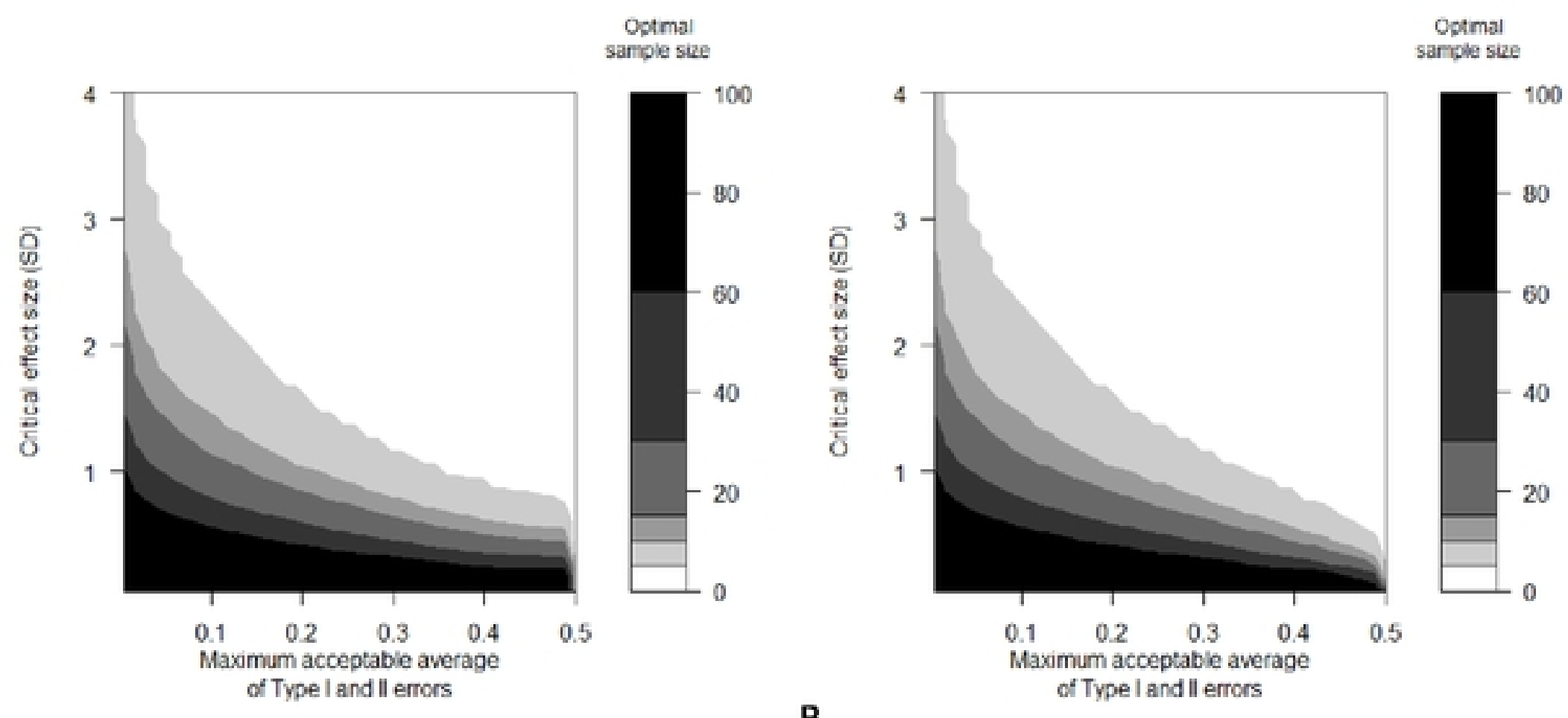

B bioRxiv preprint doi: $h$ ttps://doi.org/10.1101/540468; this version posted February 4,2019 . The copyright holder for this preprint (which was not
certified by peer review) is the author/funder, who has granted bioRxiv a license to dis displaythe preprint in perpetuity. It is made available under
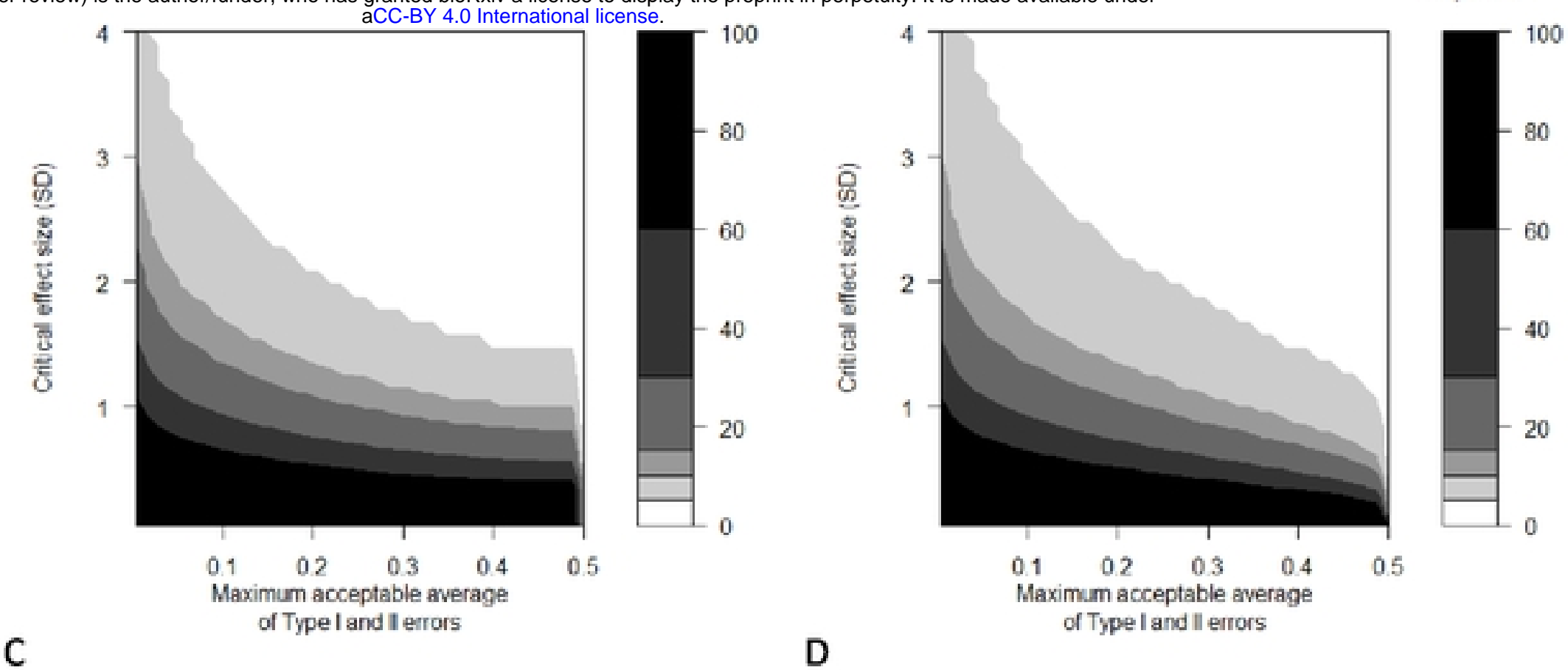

Optinal
sample size

C

Figure 5 


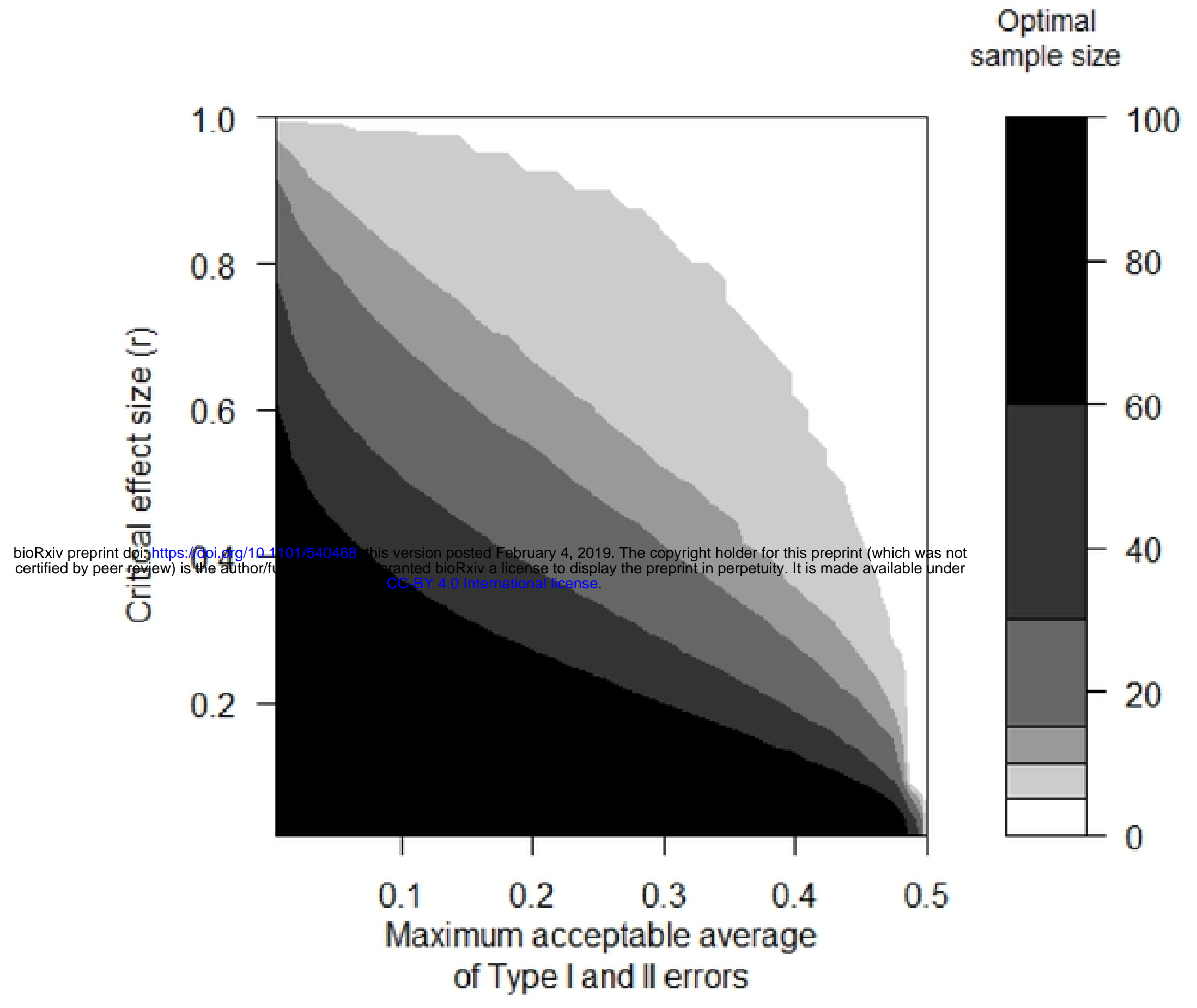

Figure 6 

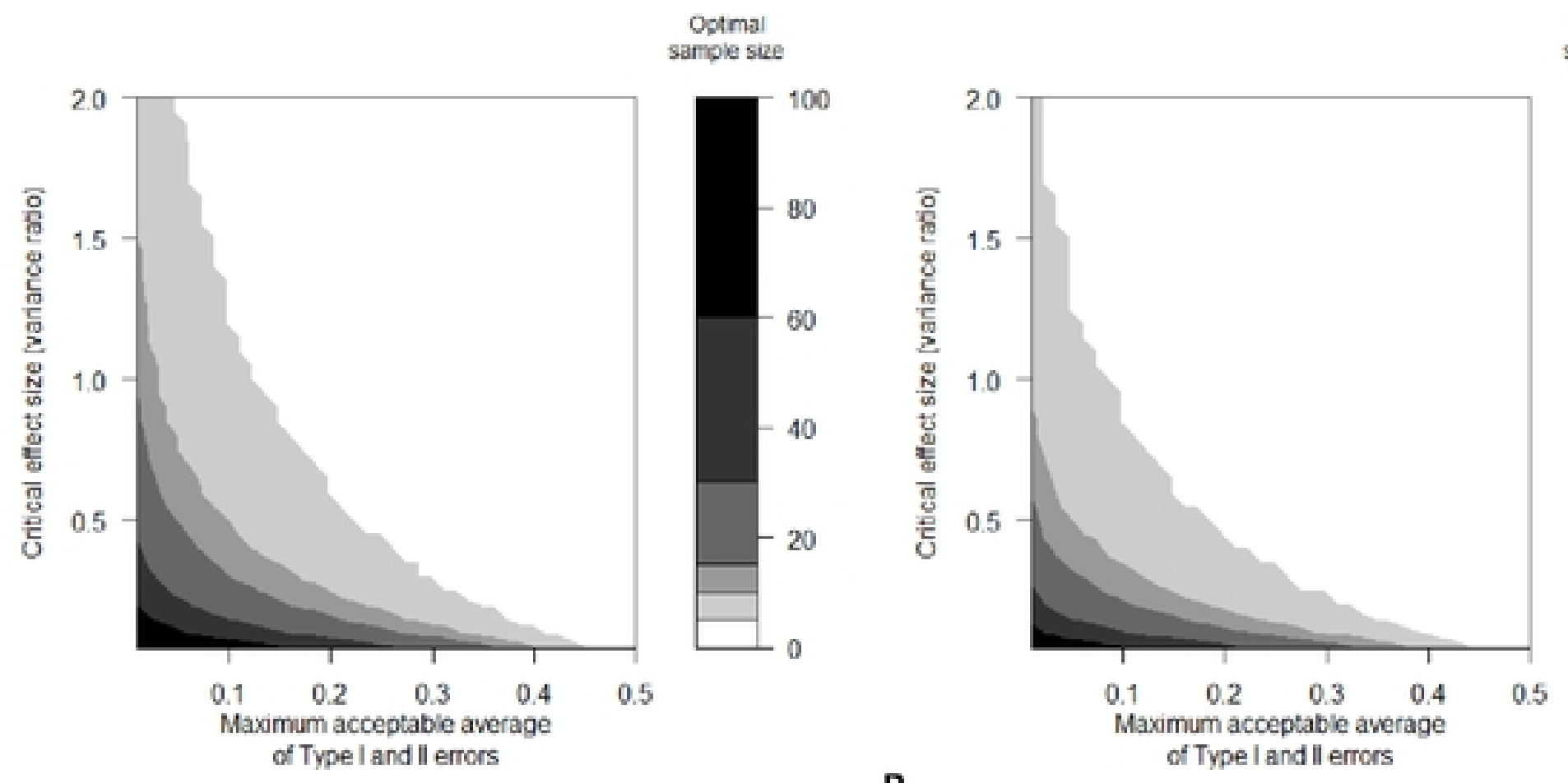

Optmal

B

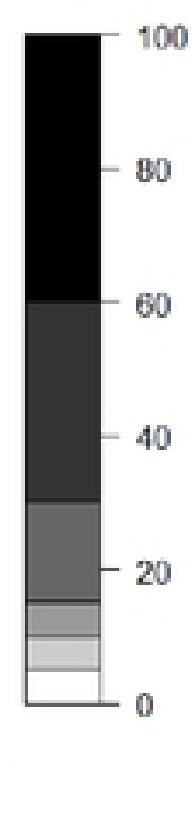

bioRxiv preprint doi: https://doi.org/10.1101/540468: this version posted February 4, 2019. The copyright holder for this preprint (which was not certified by peer review) is the author/funder, who has granted bio Rxiv a license to display the preprint in perpetuity. It is made available under
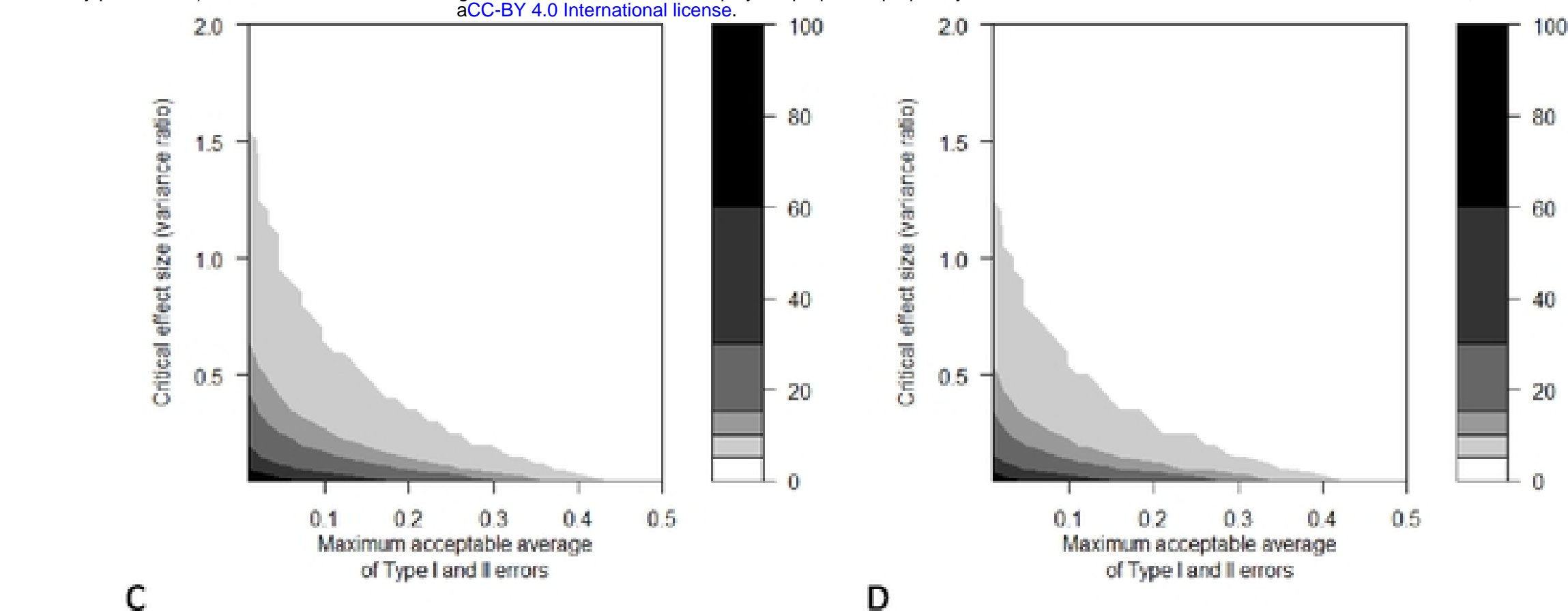

C

D

Figure 7 
Optimal

sample size

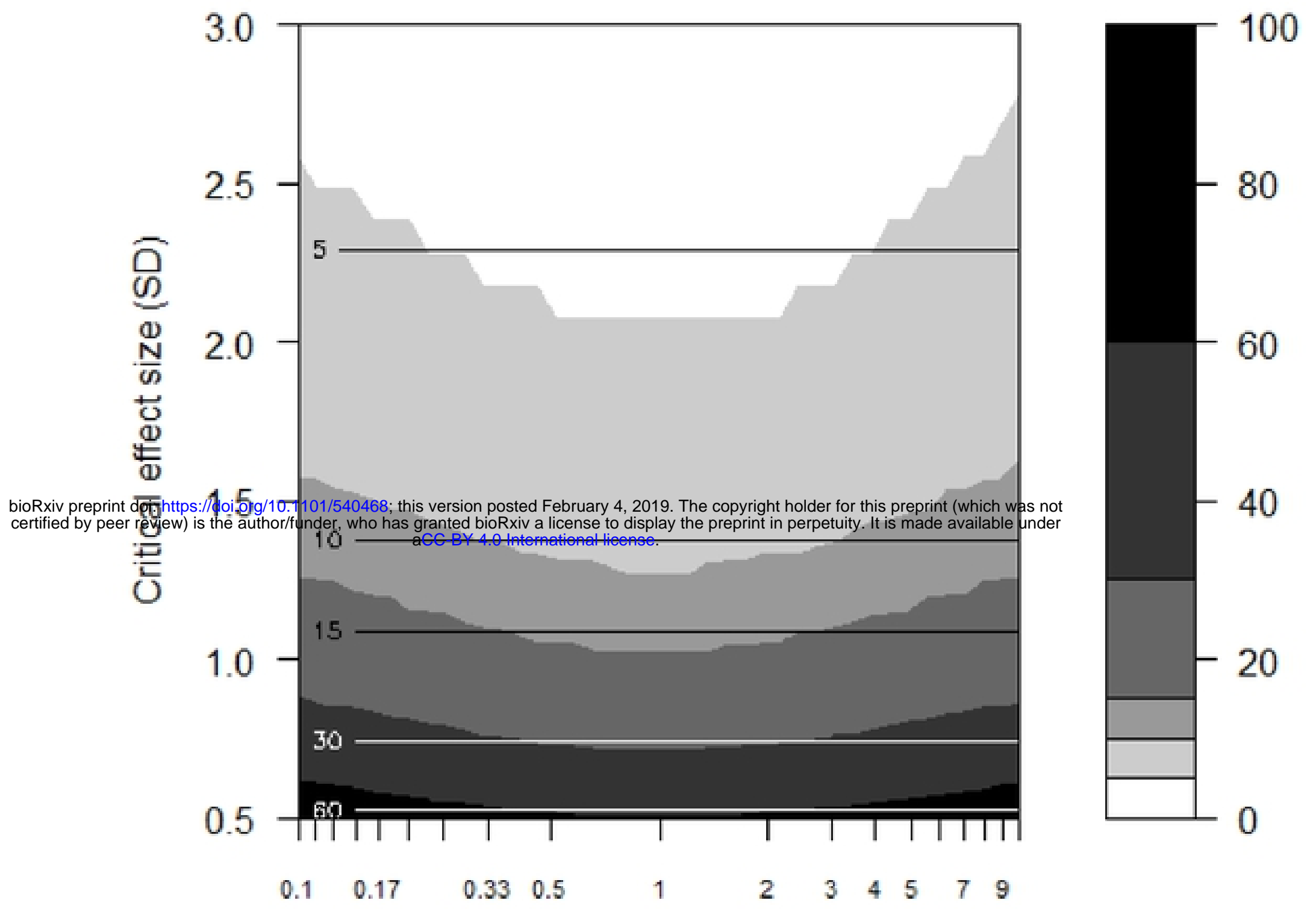

Type I vs. Type II error cost ratio

Figure 8 